R. Histórla, Săo Paulo, n. 127-128, p. 115-129, ago-dez/92 a jan-jul/93.

\title{
LE BRESIL DANS L'IMAGINAIRE FRANÇAIS (XVIè-XVIIè s.)
}

\section{Jacques Lafaye *}

\begin{abstract}
RESUMO: O autor se propóe a mostrar, $\infty \mathrm{m}$ a ajuda de exemplos empregados, a vida econômica, social e religiosa, que o Brasil ocupa no imaginário dos franceses, turante essa época, um lugar que ultrapassa largamente a imagem convencional de um exotismo tropical.

As empresas navais dos normandos, as suas empresas missionárias e bretōes dentro da obediência da Igreja Católica, as tentativas dos huguenotes perseguidos a procurarem um refógio providencial, a implementaçāo do primeiro comércio transatlântico e seus efeitos econômicas sobre a França, sto todos os aspectos de açōes inspiradas pelo "sonho brasileiro".

No campo da criaçăo artistica e da especulaçáo intelectual, a primeira imagem de uma humanidade nova e diferente foi para os franceses aquela do Indio Tupinamba, presente mesmo na França para serem atores de espetáculos póblicos. E além disso foram os primeiros cristáos batizados na corte real, os primeiros "selvagens" na visâo popular e os primeiros interlocutores americanos de um Michel de Montaigne. Os indios brasileiros, do reino de Henrique II ao do Rei Sol, foram na França os únicos representantes do Novo Mundo; foram o mesmo que a flora e a fauna do Brasil.
\end{abstract} lica.

PALAVRAS-CHAVE: imaginário, Tupinambás, bom-sejvagem, França Antártica, Igreja Cato-

Au milieu des fastes commémoratifs du Vème centenaire de la Découverte de l'Amérique, l'ombre de Christophe Colomb et de son premier Voyage aux Antilles se projette plus que jamais sur l'événement. Le Brésil, l'état de loin le plus étendu et le plus peuplé du continent Sud américain, fait figure de grand absent. Aurait-on oublie qu'il fut reconnu par Americo Vespucci comme le quatrième continent? Et que sur ta carte publiée à Saint Dié, en Lorraine, dès 1507, pour illustrer la "Nouvelle cosmographie", l'expression "Terre d'Améric" (sic) s'applique alors exclusivement au Brésil? le Brésil et sa population qui offrit à l'esprit européen ses premières images du Nouveau Monde. Ce fut au premier chef en France que le Brésil suscita un véritable branle-bas de l'imaginaire. Mais qu'il me soit permis

- Université de Paris - Sorbonne. 
LAFAYE, Jacques. Le Bresil dans l'imaginaire Français (XVIe-XVIle s.).

d'insister sur le caractère aussi actif que spéculatif d'un enthousiasme et d'un émoi si étrangers aux voiles d'abstraction (la rencontre de deux mondes, la découverte de l'Autre...) dont on enveloppe aujourd'hui la" Découverte". La "France équinoxiale" que de hardis pionniers tentèrent de bâtir en terre Brésilienne, devait être une "nouvelle France" à l'heure où l'ancienne France était meurtrie par les Guerres de Religion.

\section{NORMANDS ET TUPINAMBÁS: UNE SEULE NATION}

Longtemps avant l'entreprise de Villegagnon, les Normands avaient abordé le Brésil. C'est si vrai que dès 1503 , un gentilhomme nommé Paulmier de Gonneville avait ramené en Normandie le fils d'un cacique indien de Pernambouc, auquel il fit donner une éducation Française et légua tous ses biens. Ce fut donc par un geste magnanime et une sorte de coup de coeur que commença une longue intimité entre navigateurs Normands et indiens Brésiliens. Ceux de Honfleur semblent bien avoir fréquenté les eaux Brésiliennes dès les premières années du XVIème siècle. La recherche du profit n'était évidemment pas étrangère à ces périlleuses traversées de l'Atlantique sud. Le commerce du bois Brésil, du roucou, et aussi des aras et des singes qui devinrent à la mode, enrichirent les marchands de Rouen; la renommée du plus riche d'entre eux, Jean Ango, dépassa les frontières de la Normandie. L'Eldorado Brésilien, antérieur àla Colombie, ne fut pas un leurre, il transforma les ports normands, de Dieppe à Honfleur, en autant de "paradis économiques" avant la lettre.

Ce qui aurait pu être une opération coloniale parmi d'autres, comme celle inaugurée par Colomb à l'Ile Espagnole, qui aboutit à la dépopulation en l'espace d'une génération, fut au contraire une expérience d'acculturation réussie. Normands et Tupinambás bilingues se multiplièrent, grâce à l'amitié qui naquit et à une coexistence qui fit voir le jour à des métis, intermédiaires commerciaux parfaits entre fournisseurs indiens et marchands normands. Mais au delà de la commodité, il y eut le projet d'une société métisse. Si l'on rappelle que l'empire espagnol des Indes était fondé sur la discrimination ethnique et la ségrégation des "castas de mestizos", le rêve et la pratique sociale des Normands du Brésil a représenté un modèle alternatif de colonisation. Lorsqu'ils proposèrent à leurs alliés Tupinambás de créer avec eux "une seule nation", s'ils y avaient réussi, la face de l'Amérique en eât été changée. Ce dessein fut repris plus tard, à Recife, par Maurice de Nassau, mais il fut aussi éphémère que la présence hollandaise au Brésil. 
R. História, Såo Paulo, n. 127-128, p. 115-129, ago-dez/92 a jan-jul/93.

\section{UN SPECTACLE BRESILIEN A ROUEN}

Les marins normands avaient ramené de la région de Pernambouc une cinquantaine d'Indiens, à point nommé pour offrir un spectacle inédit au roi Henri II et à sa jeune épouse, Catherine de Médicis, récemment arrivée de Florence, en visite à Rouen. La fête consista en un combat amphibie où des pirogues s'affrontèrent sur la Seine, tandis que sur les berges et dans les fourrés d'alentour des guerriers indiens simulaient l'attaque d'un village. Comme les "Brasilians" n'étaient pas assez nombreux, quelque deux cents marins normands se déguisèrent en Indiens pour leur venir en renfort. Cet "ébastement américain", comme l'a qualifié un commentateur contemporain, fut certainement le premier du genre en Europe. Par chance une description minutieuse de la fête a été publiće à Rouen, l'année suivante (en 1551) et un graveur (anonyme mais plein de talent) l'a dépeinte avec cette légende "Figure des Brasilians".

\section{GUANABARA: TERRE PROMISE DES HUGUENOTS}

A un moment critique où l'avenir des Huguenots sembla àjuste titre menace, un de leurs chefs de file, l'amiral Gaspar de Coligny, encouragea Villegagnon à tenter de fonder une terre de refuge au Brésil. De Genève, Calvin lui-même favorisa l'entreprise et envoya un émissaire, Jean de Léry. C'est ainsi que, ayant débarqué dans l'île qui porte aujourd'hui le nom de Villegagnon, un groupe de Français fonda la première colonie européenne de la baie de Rio, qu'ils appelèrent ambitieusement la "France antarctique". Ds 1557 furent publiées à Paris les lettres de Nicolas Barré, qui évoquaient l'expédition de Villegagnon, et l'année suivante parut le livre classique du cordelier André Thevet, Les singularités de la France antarctique, autrement nommée Amérique. Ce dernier décrit l'ile, baptisée alors "de Coligny", comme: "fort plaisante pour être revêtue de grande quantité de palmiers, cèdres, arbres de Brésil, arbrisseaux aromatiques verdoyants toute l'année $(\ldots)^{\prime \prime}$; un petit paradis en d'autres termes, dont Thevet propose un inventaire des richesses naturelles et un tableau émerveillé.

Hélas cet enthousiasme, partagé par Jean de Léry, qui fit le premier l'apologie des Indiens Tupinambás dans son livre intitulé Histoire d'un voyage fait en la terre du Brésil, autrement dite Amérique, paru à La Rochelle en 1578, cet enthousiasme devant le pays de Guanabara n'empêcha pas les dissensions de renaître. Villegagnon était un catholique intransigeant, cruel même, et le contingent de Huguenots qui était à l'origine de l'expédition, 
LAFAYE, Jacques. Le Bresil dans I'imaginaire Français (XVIe-XVIIè s.).

manifesta rapidement son désaccord. Affaiblis par leurs divisions confessionnelles et sans secours de la monarchie, les Français ne purent résister, malgré l'appui de leurs alliés indiens, à l'assaut des Portugais, qui les délogèrent de l'île en 1566. Quoi qu'il en soit des infructueuses tentatives ultérieures de la France pour reconquérir sa position dans la baie de Rio, la "France antarctique" fut, bien avant l'arrivée du May Flower au cape Cod, la première colonie protestante du Nouveau Monde.

\section{L'HERBE À NICOT ET LES FUMÉES DU BRÉSIL}

Les Portugais les premiers avaient signalé la culture d'une herbe à fumer par les Tupinambás d'une presqu'île appelée Petun en tupi -d'où le verbe "pétuner" pour "fumer", qui s'imposa d'abord en Français. Le fait est que l'Ambassadeur de France à Lisbonne, Jean Nicot, rapporta au roi, en 1560 , du tabac à priser, comme un remède contre les maux de tête dont souffrait la reine-mère, Catherine de Médicis. Cette plante nouvelle reçut le nom de "herbe à Nicot" et son suc celui de "nicotine"; le nom savant du tabac, nicolina tabacum, rappelle également son premier importateur. L'usage de fumer se répandit aussi vite que celui de priser, à cause de l'impression de détente ressentie par le fumeur. Bientôt les moralistes se déchaînèrent contre cette drogue toxique, comme l'auteur du traité $\mathrm{De}$ abusu tabaci et herbae thae, paru à Strasbourg (Argentorati); le pape Urbain VIII dénonça cette nouvelle plaie venue d'Amérique... La culture du tabac se répandit cependant dans les régions chaudes, comme l'Acquitaine et, faute de pouvoir l'entraver, la monarchie institua le monopole d'Etat, sous le ministère d'un homme qui s'intếressa de près au Brésil, Colbert.

\section{L'AMI DES TUPINAMBÁS, MICHEL DE MONTAIGNE}

Les commentateurs ont voulu retenir du chapitre intitule "Des cannibales" Essais, Livre 1,ch.xxi) son aspect provocateur, l'apologie du cannibalisme qui ne pouvait que susciter l'indignation d'un lecteur qui prendrait la chose au premier degré:

"Je pense qu'il ya plus de barbarie à manger un homne vivant qu'à le manger mort (...)le faire rôtir par le menu, le faire mordre et meurtrir aux chiens (...) que de le rôtir et manger après qu'il est trépassé."

Montaigne, lecteur de Benzoni et de Chauveton (traducteur de Gomara) voire de Las Casas, voulut ici comparer la barbarie des supplices 
R. HLstórla, Sâo Paulo, n. 127-128, p. 115-129, ago-dez/92 a jan-jul/93.

infligés à des Indiens par des conquistadors accompagnés de chiens molosses, aux usages alimentaires, qui étaient également des pratiques rituelles, des Tupinambás. Avant lui Jean de Léry avait fait une réflexion analogue, mais sans en tirer un parti comparable. Ce qui est remarquable c'est la rencontre du moraliste avec des Tupinambás et leur culture. Montaigne nous apprend en effet qu'il avait eu un informateur oral, un ancien marin de Villegagnon, qui avait passé douze ans au Brésil, "un homme simple et grossier, qui est une condition propre à rendre véritable témoignage", précise-t-il. Avec un scrupule d'enquêteur ethnographique, Montaigne avait recoupé ces récits par le témoignage d'autres anciens marins de Villegagnon. Il évoque aussi le souvenir de trois Tupinambạ́s, venus à Rouen, sous le règne de Charles IX, qui s'étaient livrés à des commentaires sur le royaume de France, dignes du Huron de Voltaire, avec deux siècles d'avance! Qui plus est Montaigne avait "parlé à l'un d'eux fort long temps", tout en regrettant la sottise de l'interprè te, qui lui avait gâché le plaisir de cette conversation. De tout cela le moraliste conclut:

"Or je trouve (...) qu'il n'y a rien de barbare et de sauvage en cette nation (Tupinambá), sinon que chacun appelle barbarie ce qui n'est pas son usage."

Et l'auteur poursuit:

"Ces nations me semblent donc ainsi barbares pour avoir reçu fort peu de façon de l'esprit humain et etre encore fort voisines de leur naveté originelle", de sorte que "Nous les pouvons donc bien appeler barbares eu égard aux règles de la raison, mais non pas eu égard à nous, qui les surpassons èn toute sorte de barbarie."

Puis Montaigne fait l'éloge de leur poésie et celui, non moins audacieux que de leur cannibalisme, de leur polygamie, pour terminer le chapitre par une pointe: "Tout cela ne va pas trop mal: mais quoi, ils ne portent point de haut de chausses".

On a assez souvent fait observer que Montaigne avait posé la première pierre d'une conștruction intellectuelle que les Philosophes du XVIII ème siècle achèveraient, le "Bon Sauvage", cette machine de guerre idéologique qui devait ébranler l'autorité de l'Eglise et abattre la monarchie, en tout cas y contribuer. Plus importante encore est à nos yeux cette rencontre de Montaigne avec la civilisation Tupinambá, à cause de l'impact qu'elle eut sur 
LAFAYE, Jacques. Le Bresil dans l'imaginaire Français (XVIe-XVIle s.).

son esprit et qui n'est rien de moins que le dépassement de l'ethnocentrisme des Européens. Ce que Montaigne énonce, comme en se jouant, c'est "le principe de la relativite" des valeurs morales, ouvrant la voie à de nouveaux savoirs qui ne s'appelaient pas encore les sciences sociales et anthropologiques.

Autrement dit, le Tupinanmba de Montaigne est l'homologue de la pomme de Newton!

\section{L'EGLISE BRESILIENNE DES CAPUCINS NORMANDS ET BRETONS}

Après les marins et les marchands, la Normandie envoya au Brésil des moines évangélisateurs. En réalité le projet d'une colonisation directe prit corps en 1610, lorsque la Régente, Marie de Médicis (est-ce pur hasard si ce fut une princesse d'origine florentine?), envoya au Maragnan le sieur de la Ravardière, muni d'une patente de Lieutenant général. En même temps fut crée une compagnie coloniale (la première du genre en France, tournée vers l'Amérique?) sous l'égide du richissime Conseiller de Harley. Du point de vue qui nous occupe, trois religieux capucins accompagnèrent l'expédition; deux d'entre eux à cóté de leur labeur proprement missionnaire, écrivirent leur précieux témoignage sur le pays et sa population. Malheureusement pour les contemporains, le Voyage dans le Nord du Brésil fait durant les années 1613-1614 du P. Yves d'Evreux ne put paraître qu'à la fin du XIX siècle. Plus heureux en cela que son compagnon le $P$. Claude d'Abbeville publia, dès 1613, un opuscule qui connut un succès international immédiat, $L$ 'arrivée des Pères Capucins en l'Inde Nouvelle appelée Maragnan, (paru simultanément à Paris et à Lyon, traduit en italien et en allemand la méme année). De sorte que, dès qu'elle fut imprimée à Paris, l'année suivante, l'Histoire de la mission des Peres Capucins en l'Isle de Maragnan et terres circonvoisins (sic), de Claude d'Abbeville, fut enlevée aussitôt par les lecteurs avides d'en savoir plus sur le Maragnan; une réimpression fut nécessaire la même année.

L'ouvrage du P. Claude, tout empreint de sympathie pour les Tupinambás, décrit les "singularités admirables et les moeurs merveilleuses" de ces indiens Brésiliens. Il s'émerveille du pays, notamment de l'ile Fernando de Noronha, qu'il qualifie comme un "petit Paradis" :

"C'est un vrai pays aux oiseaux,comme de fait en ces quartiers là y a une ile appelée Fernand de la Rogne,en laquelle se trouvent tant d'oiseaux qu'on les peut (comme l'on dit) gauler ainsi qu'on fait les pommes en Normandie." 
R. Htstória, Săo Paulo, n. 127-128, p. 115-129, ago-dez/92 a jan-juj/93.

Après la mission du Maragnan, une autre fut envoyée, sensiblement plus tard dans le siècle, chez les indiens Cariris, dans la région de Pernambouc. Comme pour le Maragnan, un des religieux, un Breton cette fois, le P. Martin de Nantes, a publié son témoignage riche d'information sur le pays et les indiens, la Relation succincte et sincère de la Mission (...) dans le Brésil. La période couverte par l'auteur s'étend entre 1670 et 1700 (c'est à dire qu'elle est beaucoup plus longue que celle de Claude d'Abbeville, resté quelques mois seulement au Maragnan). Dans les deux cas, nous voyons les capucins Français se faire les protecteurs des indiens contre la cruauté des Portugais; le P. Martin écrit en effet:

"le Brésil est un exil et une retraite de plusieurs criminels (...) ce pays se remplit plus d'habitants defectueux et vicieux que d'autres, parce qu'on y vit avec beaucoup de liberté et de libertinage, et que le crime y règne assez impunément".

Il est juste de reconnaître que si les Français dénonçaient la cruauté des Portugais envers les Indiens, les Portugais accusaient les Français de paillardise à l'égard des indiennes!

Qu'elle fat une utopie éphémère (les Français, abandonnés par la monarchie, furent chassés du Maragnan par les Portugais, dès 1615), n'empecha pas l'Eglise de "l'Inde nouvelle" d'exister au Maragnan et à Pernambouc, par la vertu des religieux capucins normands et bretons.

\section{UN SUCCES PARISIEN, SIX "SAUVAGES" BRESILIENS RUE SAINT HONORE.}

Le sieur de Rasilly et le P. Claude d'Abbeville, de retour du Maragnan, firent une entrée très remarquée à Paris le 12 avril 1613. Une bonne centaine de leurs frères capucins des couvents de Paris et de Meudon, sous la houlette du Commissaire de la Province de Paris, s'en furent à leur devant, chantant un Te Deum. Puis ils se rendirent tous, en procession à l'église de leur couvent de la rue Saint Honoré qui pouvait à peine contenir "les princesses, dames et autres personnes de mérite" qui s'y pressaient, rapporte le $P$. Claude, dans son Histoire. C'est qu'ils étaient accompagnés de six indiens du Maragnan, porte-parole des caciques, "revêtus de leurs beaux plumages et tenant leur maraca à la main". Le P. Claude qui officia pendant la messe leur "fit dire le Pater noster el l'Ave Maria en leur langue, selon qu'on leur avait appris" et il ajoute ce commentaire: 
LAFAYE, Jacques. Le Bresil dans l'imaginaire Français (XVIe-XVIIe s.).

"La foule du peuple était si grande que nous fumes forcés de nous retirer avec les indiens dedans notre couvent (...) $\mathrm{Ce}$ remède fut plus propre pour altérer que pour désaltérer le désir que le peuple avait de voir ces Indiens (...) pour résister à la foule et à l'importunité du peuple, il fallut que Sa Majesté envoyat des gardes aux portes de notre couvent."

et le P. Claude de conclure:

"Mais qui eat jamais pensé que le peuple de Paris, tant accoutumé à voir des choses rares et nouvelles, se fât ému comme il a fait pour la venue de ces indiens?"

Trois des Tupinambás périrent bientôt (probablement d'affections pulmonaires dues au changement de climat), mais les survivants furent baptisés par l'évêque de Paris, en l'église des Capucins, en présence du roi et de la reine Régente, qui furent leurs parrains; les indiens "vêtus de robes de taffetas blanc et tenant à la main une fleur de lys" (les gravures qui illustrent l'Histoire du P. Claude, les représentent en ce costume) sortirent en procession avec les Capucins et l'assistance, si l'on en croit le P. Claude, "avait peine à retenir ses larmes". Les Tupinambás reçurent respectivement les prénoms de Louis-Marie, Louis-Henri et Louis de Saint Jean. Tous les trois allaient tomber malade a leur tour, mais Dieu les sauva miraculeusement, encore que l'un d'eux ait été "tenté et poursuivi par le Diable"...

On aura garde d'oublier un jeune indien Tapuya, agé de douze ans, esclave des Tupinambás, qui, tout esclave qu'il fât, devait être également baptisé, du nom de Louis-François; une noble dame et un Marquis de Courtenant furent ses parrains de baptème.

L'indien d'Amérique dans l'imaginaire parisien, et dans l'expérience, était donc encore au XVIIè siècle (comme c'était déjà le cas au XVI), l'indien du Brésil, à vrai dire c'était le seul.

\section{DE LA "FRANCE ANTARCTIQUE" AUX "SEPT TRESORS DU MARAGNAN", DE GRANDS DESSEINS AVORTES.}

Quant a ce qu'on appellerait aujourd'hui l'imaginaire géopolitique Français, il fut de bonne heure motivé par le Brésil. Dès 1543 Alphonse le Saintongeais avait publié une description du Nord du Brésil, dont il ne reste 
que des fragments. Le portulan dessiné par le normand Guillaume le Testut date de 1555. L'expédition de Villegagnon dans la baie de Guanabara, à côté de finalités économiques et religieuses, visait à disposer d'une escale fortifiée dans l'Atlantique sud. Cet enjeu était si important que les Français, délogés par les Portugais, reprirent la position, d’ô ils furent chassÉs une seconde fois, en 1561. Vingt ans plus tard, ils firent une nouvelle tentative, infructueuse, pour reprendre le fort Coligny.

La région de Bahia, d'autre part, la province de Pernambouc et plus précisément l'embouchure du fleuve Paraiba (que les capucins Français avaient appelé "le fleuve de Saint François") était une autre position clé, à la fois économique et stratégique sur la côte Brésilienne. La culture et le commerce du clou de girofle ("meilleur qu'en Indonésie"!) étaient un aspect de la concurrence à laquelle se livraient la France et les pays ibériques.

Pour ce qui est du Maragnan, il faut rappeler que Rasilly et les Capucins avaient été soutenus financièrement par des marchands parisiens. Le P. Arsène et le P. Claude Écrivirent en 1613 à l'un de ces "sponsors":

"Quant au pays, il est fort bon, et espérons d'en tirer force petun, et force roucou. Il s'y trouve dès maintenant force sucre, de fort belles pierres et de l'ambre gris, et tient-on qu'à vingt lieues d'ici il y a une mine d'or."

Si les Portugais furent si déterminés à chasser les Français du Maragnan (La Ravardière, sacrifié par la monarchie à un projet de mariage dynastique, fut incarcéré trois ans dans la tour de Belem), c'est qu'il s'agissait d'une guerre du tabac (petun), comparable à une échelle plus modeste, à la "Guerre de l'opium" en Orient.

Cet épisode ne fût peut-Être pas resté sans lendemain, si Colbert, le grand ministre de la Marine, avait vécu quelques années de plus. En effet, un de ses agents de renseignements, le sieur de Sainte Colombe, avait réussi à s'emparer (par ruse ou corruption?) d'un routier portugais, carte attribué à Teixeira et commentaire attribué à Mauricio de Heriarte. Dans une note àColbert, accompagnant ces documents confidentiels, Sainte Colombe évoque les "Sept trésors du Maragnan": le clou de girofle, la cannelle, un bois dit sampugna "propre à faire une teinture noire admirable"; il ajoute, plein de mystère, que les quatre autres trésors ne sont encore venus à la connaissance de personne.

D'autre part, d'un point de vue stratégique, l'embouchure de l'Amazone et les iles qui en étaient le verrou, pouvaient ouvrir aux Français des perspectives continentales aux conséquences incalculables sur le plan international. Qu'on en juge seulement: 
LAFAYE, Jacques. Le Bresil dans l'imaginaire Français (XVIe-XVIIe s.).

Sainte Colombe suggérait à Colbert une reconquête du Maragnan, àla faveur de la haine qu'inspirait à nos anciens alliés indiens les "Peros" (sobriquet donné aux Portugais); ce coup de main serait aisé, disait-il, en utilisant la Guyane comme base arrière. Une fois à nouveau maîtres du Maragnan, les Français pourraient remonter le cours de l'Amazone, jusqu'à rejoindre le piémont amazonien du Péreu et, là, susciter des révoltes d'Indiens pour prendre à revers le bassin des mines d'argent du haut Pérou (Potosi), qui était la réserve monétaire inépuisable de la monarchie espagnole. Un aussi vaste dessein, et son auteur, eussent mérité un sort plus enviable. Colbert venait de mourir quand ces documents parvinrent entre les mains de ses secrétaires; son successeur n'en fit rien d'utile; ils ont été retrouvés plusieurs siècles après dans la bibliothèque du prince Eugène de Savoie. Quant à l'agent de renseignements, il fut arrêté par les Portugais et finit ses jours dans la tour de Belem, où l'avait précédé le sieur de la Ravardière.

\section{LE TUPINAMBÁ DU MARAGNAN, ANCETRE DU "BON SAUVAGE"}

Si les chapitres des Essais de Montaigne consacrés au Nouveau Monde, et plus encore le chapitre Des cannibales inspiré par sa rencontre avec des indiens du Bresil, ont fait l'objet de nombreux commentaires, voire d'interprétations discutables, il n'en va pas de même pour l'Histoire de Claude d'Abbeville. Cela tient à la richesse, à la variêté et à la portée philosophique et morale de l'immortel ouvrage de Montaigne, sans doute. Si l'on y regarde de plus près cependant, on remarque que l'Histoire du P. Claude fut saisie dès 1615 et celle du P. Yves (qui en était la suite) lacérée sur ordre royal; celà pour convenance politique du moment. Un livre capital pour la connaissance du Brésil et de sa population autochtone disparut donc au bout d'un an et pour très longtemps (il fallut aller chercher un des rares exemplaires complets à la bibliothèque de Göteborg, pour en faire la réédition fac simile, en 1964 ). Or le livre du P. Claude est beaucoup plus riche d'information sur les Tupinambás, que le chapitre de Montaigne, pour la bonne raison que le Capucin avait passé plusieurs mois au Maragnan, parmi les Indiens. Qui plus est sa réflexion, moins provocante, est plus riche que celle de Montaigne car elle s'applique à tous les domaines d'une culture, dont Montaigne n'avait pu entrevoir que quelques traits surprenants ou choquants pour ses contemporains. La genèse des idées du capucin normand sur les "Brésiliens" et de son regard sur eux, mérite qu'on s'y arrête un instant. Il nous apprend lui-même que sur la foi de ses lectures (peut -être 
R. Hlstórla, Såo Paulo, n. 127-128, p. 115-129, ago-dez/92 a jan-jul/93.

Jean de Léry, ou même Staden?), il avait débarqué au Maragnan la tête pleine de "méchants sauvages nus, terribles mangeurs d'hommes". Or ayant observé les Tupinambás dans leur existence quotidienne, véritable chasseur d'images autant que pêcheur d'âmes, ses yeux s'ouvrirent, la crainte se muá en sympathie. Il les dépeint robustes et valiđes, "allègres et dispos"; leur vie est saine et leur beauté physique en est l'effet. Leur nudité est la marque de leur innocence:

"Je puis dire qu'il y a sans comparaison beaucoup moins de danger à voir la nudité des Indiennes que la curiosité des attraits lubriques des dames mondaines de la France."

Le P. Claude brosse de la société Tupinambá un tableau idyllique: l'entente des couples est remarquable; les parents ne crient jamais sur les enfants; les divorces s'ils surviennent se règlent sans conflit; les épouses du polygame s'entendent bien entre elles; la polygamie est justifiée par la forte mortalité masculine à la guerre et à la chasse; l'amour maternel et l'amour filial y sont très forts; l'amitié y est sacrée et la solidarité du groupe est le ciment de la société Tupinambá.

Sur ce fond d'harmonie parfaite, le capucin moraliste autant qu'ethnographe aborde la question capitale de l'élevage des bébés: on les frotte d'huile et on les met à dormir à l'air, dans des hamacs où ils respirent; on se garde de les emmailloter et c'est pour cela qu'ils ne sont jamais contrefaits, comme les enfants Français" enserrés dedans leurs berceaux et toute leur vie dans des accoutrements si étroits, que la nature étant comme prisonnière et violentée (...)" On croirait lire L'Emile et c'est Claude! Il ajoute que les indiennes allaitent elles-mêmes leurs enfants "elles n'ont garde de les donner à des nourrices", ainsi de suite. L'indien du P. Claude (ici l'indienne) est donc à l'évidence le prototype du "Bon sauvage" des Lumières, même si ce fut à travers des auteurs intermédiaires.

Cela serait très suffisant pour la gloire du capucin normand, mais ce n'est pas tout. L'arrivée des Tupinambás à Paris fít grand bruit, jusqu'en province. Malherbe reçut une lettre de Fabri de Peiresc et s'intéressa à la langue tupi. Boileau citera plus tard Claude d'Abbeville (preuve que les deux tirages de l'Histoire, vendus avant la saisie, étaient encore accessibles dans les bibliothèques particulières). Descartes avait dix huit ans en 1614 et on sait qu'il dévorait des livres; comment ne pas penser à l'axiome essentiel du Discours de la méthode, en lisant Claude d'Abbeville:

"Ils sont fort raisonnables et ne se laissent conduire que par la raison, et non sans connaissance de cause" 
LAFAYE, Jacques. Le Bresil dans l'imaginaire Français (XVIe-XVlle s.).

et encore ceci :

"les Maragnans (...)sans lecture, sans études, ont néanmoins l'esprit et le jugement naturel aussi bon qui se puisse trouver"

Autant dire qu'ils sont "l'homme cartésien". Où diable Descartes aurait-il bien pu aller chercher l'idée que "le bon sens est la chose du monde la mieux partagé", c'est à dire (en Français moderne) que la capacité de jugement est équitablement répartie dans l'humanité toute entière? - et non réservée aux "civilisés", comme l'avait prétendu Aristote, duquel Descartes voulut précisément saper l'autorité. On saisit sur le vif comment le surgissement du rationalisme moderne procède directement de ces precurseurs de l'ethnographie que furent les missionnaires, au moins les plus doués et les plus ouverts d'entre eux, comme le P. Claude.

\section{EPILOGUE}

Il seraił difficile de proposer une conclusion à cette série d'évocations, mais on aperçoit sans qu'il soit utile d'y insister trop, comment ces fils, épars en apparence, se rejoignent et se nouent en profondeur. Profondeur historique qui fait de la "France Equinoxiale" la première "France d'Outre mer". Remarquons d'ailleurs que le Brésil imaginé, sinon durablement réalisé, par les Français des XVIè et XVIIè siècles, embrassait le Brésil côtier dans toute sa dimension moderne; telle fut la clairvoyance des marins et des missionnaires normands. Profondeur philosophique d'une portée durable, puisqu'à travers le premier regard ethnographique sur l'indien Brésilien (longtemps avant les Iroquois et les Hurons) quelques uns des plus significatifs penseurs de langue Française, Montaigne, Descartes, Rousseau, ont ouvert des voies nouvelles à la réflexion sur le genre humain.

Panacée économique, terre de refuge, terre de mission, société idéale, paradis terrestre... c'est tout cela ensemble et tour à tour que fut le Brésil dans l'imaginaire Français, et donc européen à travers celui-ci, à l'aube des temps modernes. Le Tupinambá du Maragnan est devenu sans le savoir l'image paradigmatique d'une humanité pour la première fois unifiée... idéalement s'entend. En France le Nouveau Monde américain se confondit aux origines avec les paysages, les richesses, les plantes et les animaux du Tropique brésilien, les Indiens du Brésil. L'exotisme qui a tenu une si grande place dans notre littérature et nos arts plastiques, notre alimentation et nos rêves, en un mot notre culture, est né de la rencontre des Français avec te Brésil. 
R. Htstórla, Săo Paulo, n. 127-128, p. 115-129, ago-dez/92 a jan-jul/93.

\section{RÉFÉRENCES BIBLIOGRAPHIQUES}

ABBEVILLE, Claude d', R. P. Capucin. Histoire de la mission des Peres Capucins en l'isle de Maragnan et terres circonwoisins. Paris, 1614. (ed. fac simile el introduction par Alfred METRAUX et Jacques LAFAYE, Graz, 1963)

ALCEDO, Antonio de. Diccionario geografico-histórico de las Indias Occidentales o América. Madrid, 1788. (t. II, pp. 500-503)

BATAILLON, Marcel. "Montaigne et les conquérants de 1'or", Studi francesi $\mathbf{n}^{\circ}$ 9, Turin, 1959.

BRY, Théodore de. Narratio profectionis Joanis Lerii Americae tertiae pars. Francfort, 1592.

CHINARD, Gilbert. L'exotisme américain dans la littérafure Françaisse au XVIe siècle. Paris, 1911.

DENIS, Ferdinand. Une fête Brésilienne célébrée a Rouen en 1550. Paris, 1850.

DUPUICH, Henri. "Un précurseur de la littérature exotique. Le R. P. Claude d'Abbeville (...)", Bulletin de la société d'émulation historique et littéraire d'Abbeville, t. XVII, 1942.

EVREUX, Yves. Voyage dans le nord du Brésil fait durant les années 1613 et 1614, préfacé et édité par Ferdinand DENIS, Paris et Leipzig, 1864.

FENELON, Paul, "Les apports du continent américain dans le domaine végétal et animal de l'ancien continent", in La découverte de l'Amérique, Paris, 1968 (Xè stage international d'Études humanistes, Tours, 1966), pp. 253-266.

FERNANDES, Flotestan. A organização social dos Tupinambá. Sáo Paulo, 1948.

. "Guerre et sacrifice humain chez les Tupinambás", Journal de la Société des Americanistes, t. XL, Paris, 1952.

FLETSCHAMANN, Ulrich ef al., "Os Tupinambá: realidade e fioção nos relatos quinhentistas", Revista Brasileira de História, vol. 11, no. 21, 1990-1991.

GARCIA, Rodolpho, "Glosario das palavras e phrases da lingua tupi contidas na Histoire (...) do Padre Claude d'Abbeville", Revista do Instituto Histórico Brasileiro, 1. 94, vol. 148, Rio de Janeiro, 1927.

GIUCCI, Guilhermo, "A visfo inaugural do Brasil: a terra de Vera Cruz", Revista Brasileira de História, vol. 11, no. 21, 1990-1991.

HERIARTE, Mauricio de, Descriçam do Estado do Maranham, Para, Corupa, Rio das Amazonas, Graz, 1964. (ed.fac simile de Karl Nowotny).

LAFAYE, Jacques, "La "Terre de Brésil" disputée (Rivalité entre Français et Portugais aux XVlè et XVIIè siècles)", in Arquivos do Centro Cultural Português, XXIII, Fundaçao Calouste Gulbenkian, Lisboa-Paris, 1987. pp.915-930.

LASTRE, de..., Histoire véritable de ce qui s'est passé de nouveau entre les Français et les portugais en l'isle de Maragnan au pays des Toupinambous, Paris, 1616.

LE GENTIL, Georges. "Un document inédit sur la seconde expédition des Capucins au Maranhao en 1614", Revue d'hisfoire de France, I.II, Paris, 1925.

La France Equinoxiale, Universidade de Coimbra, 1933.

LEITE DE FARIA, Francisco (OFM). Os primeiros missionários do Maranhä. A chegada para a historia dos Capuchinos franceses que at estiveram do 1612 a 1615 . As comemoraçōes enriquinas, Lisboa, 1961. 
LAFAYE, Jacques. Le Bresil dans l'imaginaire Français (XVIè-XVlle s.).

LERY, Jean de. Histoire d'un woyage fait en la terre de Brésil. La Rochelle, 1578. (Paris, 1880, ed. de Paul Gaffarel). Reéd. Lausanne, 1972 et Genève, 1975.

LESTRINGANT, Frank, "Genève et l'Amérique à la Renaissance: le rêve du refuge huguenot (1555-1600)", in America in european consciousness (May, 1991), The John Carter Brown Library . (à paraître)

LEXICON Capuccinum historico-bibliographicum Ordinis Fratrum Minorum Capuccinorum, Brundisi, 1951.

MARCEL, Gabriel. "Le Père Yves d'Evreux", Journal de la Sociêté des Américanistes, nouvelle série, vol. IV, Paris, 1907.

MARTIN DE NANTES. Relation succincte et sincère de la mission du P..., Prédicateur capucin, missionnaire dans le Brezil parmy les Indiens appelés Cariris, Quimper, 1706.

MASSA, Jean Michel. "Le monde luso-Brésilien dans la joyeuse entrée de Rouen", in Les fétes de la Renaissance, 1. III, Paris, 1875. pp.105-116.

METRAUX, Alfred. "The Tupinamba", in Handbook of South American Indians, vol. III, Bulletin 143, The Smithsonian Institution, Washington DC, 1948.

"Les preccurseurs de l'ethnologie en France, du XVIè au XVIIle siècle", Cahiers d'histoire mondiale, VII, 3, UNESCO, Paris, 1963.

MOCQUET, Jean. Voyages en Afrique, Asie, Indes orientales et occidentales. Paris, 1617.

PEZIEU, Monsicur de. Brief recueil des particularitez contenues aux lettres envoyeés par Monsieur de Pezieu a messieurs ses parents et amis de France, Lyon, 1613.

ROMUALDUS, R. P. a Santo Marcello (OFM), Notae de ignoto manuscripto versionis italicae Claudii ab Abbeville, Collectanea Franciscans, XIII, 1943.

STRAUBING, Ulrich Schmidel von. Ulrich, Warhaffige Historien einer wunderbaren Schiffahrt (...) von anno 1534 bis anno 1554 in Americam oder NeueWelt bei Brasilia und Rio de la Plata getan. Nurnberg, 1602. (ed fac simile, Graz, 1962).

STADEN, Hans. Warhaftige Historia und Beschreibung einer Landtschaft der wilden nacketen grimmigen Menschenfresser Leuthen in der Newenwelt America gelegen. Marburg, 1557.

STREIT R. (OMI). Bibliotheca Missionum. Mânster-Aachen, 1916-1930.

THEVET, André. Les singulariés de la France antanctique, Paris, 1878. (ed. de Paul Gaffarel). Réed. fac simile de l'éd. otiginale de 1557-58, Paris, 1982.

VAUCHERET, Etienne. "Jean Nicot et l'entreprise de Villegagnon", Paris, 1968, in Dixième stage international d'études humanistes. Tours, 1966; pp.89-104).

VIVEIROS de CASTRO, Augusto Olympo, "Os Franciscanos no Maranhāo", Revista do Instituto Histórico e Geografico Brasileiro. Rio de Janeiro, 1927.

ABSTRACT: The author intencts to show, with the help of examples used in the coonomic, social, and religious environments, that Brazil occupies in the French imaginary, during this time period, a level which surnasses the conventional image of an exotic tropical.

The naval entreprises of the Normancts, their enterprises of missionaires and Britons obedient to the Catholic Church, the attempts of the Huguenots to search for a providential refuge, the implementa- 
R. Hist6ria, Săo Paulo, n. 127-128, p. 115-129, ago-dez/92 a jan-jul/93.

tion of the first transatlantic commerce and its effects on the French economy are all aspects of actions inspired by the "Brazilian dream".

In the field of artistic creations and intellectual speculation, the first image of a new and different bumanity was; for the French, that of the Tupinambal lodians, present even in France to become actors of public shows. Furthermore, they were the first Christians baptized in the royal court, the first "savages" in the popular vision and the first Native American speakers of Michael de Montaigne. The Brazilian indians, from the reign of Henry 11 to the King Sun, were in France the only representadves of the New World; they were the same as the flora and fauna of Brazil.

KEY-WORDS: imaginary, Tupinambás, "good savage", France Antarctica, Catholic Cburch. 